Ciencia y Salud, Vol. II, No. 3, septiembre-diciembre, 2018 • ISSN: 2613-8816 | E-ISSN: 2613-8824

DOI: https://doi.org/10.22206/cysa.2018.v2i3.pp35-40

\title{
SÍNDROME DE TURNER Y EMBARAZO ESPONTÁNEO: PRESENTACIÓN DE DOS CASOS
}

\author{
Turner syndrome and spontaneous pregnancy: Presentation of two cases
}

\author{
Dr. Francisco Carvajal Martínez*, Dra. Danexy Borges Carcases**, \\ Dra. Yisel Ávila Baltazar**, Dra. Alicia Burgos Cabrera***, Dra. Yuraimi Piz Ramos***
}

Recibido: 24 marzo, 2018 Aprobado: 6 junio, 2018

Cómo citar: Carvajal Martínez F, Borges Carcases D, Ávila Baltazar Y, Burgos Cabrera A, Piz Ramos Y. Síndrome de Turner y embarazo espontáneo: presentación de dos casos. Ciencia Y Salud [Internet]. 14sep.2018 [citado 14 sep.2018]; 2(3):35-0. Available from: https://revistas.intec.edu.do/index.php/cisa/article/view/1281

\section{Resumen}

Introducción: El Síndrome de Turner constituye la única monosomía completa compatible con la vida. Se produce por la no disyunción meiótica o mitótica de los cromosomas, con expresión variable, que se presenta en el sexo femenino y en el que es extraordinariamente infrecuente el embarazo espontáneo.

Objetivo: Describir dos casos clínicos con diagnóstico de Síndrome de Turner que lograron embarazo espontáneo.

*Profesor titular y consultante de Endocrinología. Investigador titular y de mérito Departamento de Endocrinología Pediátrica. Instituto Nacional de Endocrinología. La Habana, Cuba.

**Residentes de 3er año de Endocrinología Hospital Clínico Quirúrgico Juan Bruno Zayas (Santiago de Cuba).

**Residente de 3er año de Endocrinología del Instituto Nacional de Endocrinología (INEN). La Habana, Cuba.

${ }^{* * *}$ Especialista 1er grado en Medicina General Integral. Residente de 2do año de Endocrinología del Instituto Nacional de Endocrinología. La Habana. Cuba.

Email: endoped@infomed.sld.cu
Presentación de los casos clínicos

Caso 1: Paciente femenina que a los 9 años de edad cronológica es remitida al Departamento de Endocrinología Pediátrica del Instituto Nacional de Endocrinología, por presentar retraso del crecimiento y desarrollo, donde a través de las manifestaciones clínicas y de los exámenes complementarios realizados, se le diagnosticó un Síndrome de Turner 45X/46XX. Recibió tratamiento con hormona de crecimiento biosintética y hormonas sexuales; logrando embarazo espontáneo a los 23 años de edad, y actualmente, tiene una hija de un ańo y medio.

Caso 2: Paciente femenina que a los de 9.4 años de edad cronológica es remitida al Departamento de Endocrinología Pediátrica del Instituto Nacional de Endocrinología por presentar retraso del crecimiento y desarrollo, donde a través de las manifestaciones clínicas y de los exámenes complementarios realizados, se le diagnosticó un Síndrome de Turner 45X. Recibió tratamiento con hormona de crecimiento biosintética, presentó menarquía espontánea a los 13 años con ciclos menstruales regulares; logró gestación espontánea a los 20 ańos, interrumpiendo el mismo por decisión personal. 


\section{Dr. Francisco Carvajal Martínez, Dra. Danexy Borges Carcases, Dra. Yisel Ávila Baltazar, Dra. Alicia Burgos Cabrera y Dra. Yuraimi Piz Ramos}

Conclusiones: El embarazo en el Síndrome de Turner es infrecuente. Existe solo una publicación nacional, y muy pocas internacionales, de pacientes con esta afección que logren gestación espontánea. Se plantea que puede existir una línea celular a nivel de ovarios y útero que permite el desarrollo folicular y así lograr la fecundación. En la actualidad las expectativas de fertilidad con este síndrome han cambiado en los últimos años, gracias a los tratamientos de reproducción asistida.

Palabras clave: Síndrome de Turner, monosomía completa, embarazo espontáneo.

\section{Abstract}

Introduction: Turner Syndrome is the only complete monosomy compatible with life. It is disjunction of chromosomes, with variable expression, it occurs in the female sex and it is extraordinarily uncommon in spontaneous pregnancy in this patient.

Objective: To describe two clinical cases with diagnosis of Turner Syndrome that achieved spontaneous pregnancy.

\section{Presentation of clinical cases:}

Case1: A female patient who, at 9 years of age, is referred to the Department of Pediatric Endocrinology of the National Institute of Endocrinology for delayed growth and development, where through the clinical manifestations and complementary examinations performed, she was diagnosed with Turner Syndrome 45X/46XX. She was treated with biosynthetic growth hormone and sex hormones; who achieved spontaneous pregnancy at age 23 and currently has a daughter a year and a half.

Case2: A female patient who is chronologically 9,4 years of age is referred to the Department of Pediatric Endocrinology of the National Institute of Endocrinology because of the delay in growth and development where, through clinical manifestations and complementary examinations, she was diagnosed with Turner Syndrome 45X. Received treatment with biosynthetic growth hormone, presented spontaneous menarche at 13 years with regular menstrual cycles; spontaneous gestation al 20 years interrupting the same by personal decision.

Conclusions: Pregnancy in Turner's Syndrome is not frequent. There is one national and very few international publications of patients with this condition that achieve spontaneous gestation. It is suggested that there may be a cell line at the level of ovaries and uterus that allows follicular development and this archive fertilization. At presents, the expectation of fertility with this syndrome has changed in the last year, thanks to the treatments of assisted reproduction.

Keywords: Turner Syndrome, complete monosomy, spontaneous pregnancy

\section{Introducción}

En 1930, el pediatra alemán Otto Ullrich describió a varios pacientes con los síntomas característicos de un síndrome al que, en 1938, Henry Turner, en un estudio de siete pacientes en EE.UU., dio este nombre. De ahí que en Europa el síndrome se conozca también como de Ullrich-Turner ${ }^{18,10}$. El Síndrome de Turner (ST) es causado por la ausencia parcial o total de un cromosoma X, provocando anormalidades somáticas características y falla en el desarrollo ovárico conocido como disgenesia gonadal. Como consecuencia de esto, las pacientes presentan amenorrea con ausencia de desarrollo de caracteres sexuales secundarios e infertilidad. Si bien lo anterior corresponde a lo clásicamente descrito, existe un $30 \%$ de mujeres ST que logran sin tratamiento, algún grado de desarrollo puberal; un $10 \%$ presenta menarquia y $2-5 \%$ tienen embarazo en forma espontánea $^{17,6}$. Afecta aproximadamente alrededor de 2,500 a 5,000 nińas en todo el mundo ${ }^{2,10,15}$.

Este síndrome es una de las anomalías cromosómicas más frecuentes, afectando el 3\% de los fetos femeninos; la anomalía 45X constituye una de las causas más frecuentes de abortos espontáneos. Con síntomas menos evidentes se encuentran los mosaicismos, siendo el más frecuente 45X/46XX ${ }^{2,5,12,15,19}$.

La expresión fenotípica del ST se puede explicar por los siguientes fenómenos:

a) el estado de haploinsuficiencia de genes, que en condiciones normales se expresan en los dos cromosomas sexuales y que escaparían al proceso de inactivación; 
b) la ausencia de dos cromosomas sexuales normales antes de la inactivación de un cromosoma X;

c) fenómenos de impronta que modifican la expresión de determinados genes en función de su procedencia, materna o paterna;

d) la aneuploidia y las consecuencias derivadas del desequilibrio cromosómico. Los estigmas somáticos se asocian con la haploinsuficiencia de genes localizados en el brazo corto del cromosoma $\mathrm{X}$, en la región distal. Tanto el brazo largo como el corto del cromosoma $\mathrm{X}$ contiene genes esenciales para la función ovárica, y la aneuploidia celular puede producir una reducción en el número y supervivencia de los oocitos. La pérdida intersticial o terminal de partes del Xq puede producir talla baja y fallo ovárico ${ }^{18,15}$.

Clínicamente se manifiesta por retraso del crecimiento en un $90 \%$ de los casos, epicantos, boca de pez, paladar ojival, implantación baja del pabellón auricular, cuello en esfinge, implantación baja del cabello. Un signo característico presente en estas niñas es el ensanchamiento relativo del tórax y de los hombros, denominado tórax en escudo, teletelia, acortamiento del cuarto metacarpiano, infantilismo sexual, presencia de manifestaciones dermatológicas dadas por nevus, vitíligo, alopecia y psoriasis. La mayoría de las pacientes con ST no tienen retraso mental, aunque pueden existir trastornos de aprendizaje, sobre todo en lo que se refiere a la percepción espacial, coordinación visual-motora y matemáticas ${ }^{2,18,19,14,12}$.

El desarrollo gonadal y el número de oocitos son normales hasta las 18 semanas en las niñas con ST. Luego comienza una pérdida acelerada y prematura de estos, cuya causa aún es desconocida. La mayoría de las pacientes con evidencias de función ovárica corresponden a mosaicos ${ }^{2,17,19}$.

Tradicionalmente las pacientes con ST se han considerado infértiles. Si bien un porcentaje logra algún grado de función gonadal, eso no significa que puedan lograr un embarazo con éxito. Esto queda demostrado por el bajo porcentaje de embarazos espontáneos y mal pronóstico de estos. Existe una alta tasa de aborto espontáneo (29\%), mortinatos $(7 \%)$ y malformaciones fetales $(20 \%)^{17}$.

Por el interés clínico que despierta y lo infrecuente de su aparición consideramos importante presentar estos casos.

\section{Presentación delos casos clínicos}

\section{Caso 1}

Paciente L.L.M, HC: 500813, producto de un parto eutócico, peso natal 4.81b. Desde el 2do año de vida se comienza a estudiar por retardo del crecimiento y alteraciones fenotípicas que fueron apareciendo a lo largo de su desarrollo psicomotor. A los 10 ańos se le diagnostica Mosaico Turner 45X/46XX remitiéndose al servicio de endocrinología pediátrica. A los 13 años acude a consulta, constatándose al examen físico, implantación baja de pabellones auriculares, paladar ojival y cuello corto.

Adrenales: Tanner III y Mamas Tanner III.

Peso: $33 \mathrm{~kg}$ Talla: $127 \mathrm{~cm}$. T/E: $<3$ percentil P/T: $>97$ percentil P/E: 25- 50 percentil

Complementarios:

- FSH: 7.4 UI/L

- LH: $4.5 \mathrm{UI} / \mathrm{L}$

- PRL: 384mUI/L

- TSH: $4.63 \mathrm{muI} / \mathrm{L}$

- T4: $141 \mathrm{nmol} / \mathrm{l}$

- Cariotipos: 45X/46XX, 45X (12 metafases), 45X/47XXX

- U/S renal: normal. 


\section{Dr. Francisco Carvajal Martínez, Dra. Danexy Borges Carcases, Dra. Yisel Ávila Baltazar, Dra. Alicia Burgos Cabrera y Dra. Yuraimi Piz Ramos}

- U/S ginecológico: Útero normal. OD folículo $12 \mathrm{~mm}$. OI pequeños folículos.

- Test de sensibilidad a la insulina midiendo GH y glucemia normal.

A partir de los 13 años recibió tratamiento con hormona de crecimiento biosintética hasta los 17 años, alcanzando una talla final de $135 \mathrm{~cm}$ con cierre del cartílago epifisario y tratamiento sustitutivo, con hormonas sexuales desde los 14 años hasta los 17. Presentó menarquía a los 14 años de edad con ciclos menstruales regulares.

Todo el tiempo creció en la línea del 3er percentil y alcanzó un desarrollo intelectual normal. A la edad de 17 años por decisión familiar no asistió a ninguna otra consulta hasta el 2016, una vez citada, a propósito de una investigación, se embarazó a la edad de 23 ańos y actualmente tiene una hija de un ańo y medio, con peso al nacer de $2700 \mathrm{~g}$, producto de un parto distócico causado por desproporción cefalopélvica.

\section{Caso 2}

Paciente femenina B.E.G, HC: 500435, producto de un parto distócico por cesárea anterior a las 41 semanas, con peso al nacer de 6.8 libras, talla: $47 \mathrm{~cm}$, circunferencia cefálica: $34 \mathrm{~cm}$, apgar $9 / 9$, caída del cordón umbilical a los 5 días de nacida. Que no presentó complicaciones prenatales ni postnatales. Recibió lactancia materna exclusiva hasta los 8 meses de edad. A la edad de 9 años es remitida para estudio al servicio de endocrinología pediátrica, por síndrome de baja talla. Al examen físico en nuestra institución encontramos presencia de estigmas turnerianos dados por epicanto, implantación baja del pabellón auricular, implantación baja del cabello, labio superior fino, paladar ojival, tórax ancho con presencia de teletelia, acortamiento del cuarto metacarpiano en ambas manos.

Peso: 19kg Talla: $111 \mathrm{~cm}$ Talla/Edad : $<3$ percentil; Peso/Edad: <3percentil; Peso/Talla:50-70percentil.
Brazada: $105 \mathrm{~cm}$, DBA: $18 \mathrm{~cm}$, DBT: $13,5 \mathrm{~cm}$, PP: $55 \mathrm{~cm}, \mathrm{CC}: 57 \mathrm{~cm}$

Tiroides: grado 0

Chvosteck: grado: I

Gónadas: Femeninas

Adrenal: Vello Púbico: Tanner: I. Vello Axilar: ausente.

Complementario

Cariotipo: 45X.

Test de sensibilidad a la insulina midiendo Glucemia y GH: Normal

Edad Ósea: 10.9años. Normal para edad cronológica.

FSH: $5.6 \mathrm{muI} / \mathrm{ml}$.

LH: $1.6 \mathrm{muI} / \mathrm{ml}$.

Estradiol: $68.59 \mathrm{pg} / \mathrm{ml}$.

Ultrasonido ginecológico: Útero tamaño normal, anejo izquierdo que mide $24 \times 12 \mathrm{~mm}$, anejo derecho mide $33 \times 14 \mathrm{~mm}$, presencia de folículos. Hipoplasia de ovario izquierdo en relación con el derecho.

Paciente que se le diagnóstica ST a la edad de 9 años, que presentó menarquia espontánea a los 13 años de edad, con ciclos regulares. Recibió tratamiento con hormona de crecimiento biosintética hasta los 14 años por cierre de cartílago epifisiario. Logró talla definitiva $143 \mathrm{~cm}$. Actualmente tiene 20 años de edad, estudia Licenciatura en Arte en la universidad, presenta estudios actuales de hormonas sexuales en valores normales (FSH: $2.5 \mathrm{mul} / \mathrm{ml}$, LH: $1.5 \mathrm{muI} / \mathrm{l}$ y estradiol: $68.59 \mathrm{pg} / \mathrm{ml}$. Estuvo embarazada con gestación de 9 semanas, interrumpió el embarazo por decisión personal para no afectar sus estudios. 


\section{Discusión}

Es conocido que no todos los casos de ST tienen un cariotipo $\mathrm{XO}$, del mismo modo que no todas las disgenesias gonadales cursan con ST. Esta afección puede cursar con alteraciones cromosómicas, pero sí con variada constitución, de las cuales la más frecuente es la XO.

Botella plantea que estas pacientes en la época prenatal tendrían ovarios perfectamente desarrollados, los cuales secundariamente se irían atrofiando de forma progresiva, hasta quedar convertidos en cintillas sin estructuras ${ }^{4,3}$.

Las posibilidades de embarazos espontáneos de estas mujeres oscilan desde el $1 \%$, para las que presentan 45X en todas sus células; a un 25\%, para las que tienen una línea celular $46 \mathrm{XX}$, a pesar de tener periodos fértiles cortos. De manera global, en las mujeres con ST las posibilidades de embarazo se sitúan alrededor del 5\% $\%^{11,9}$.

Algunos autores han publicado pocos casos con ciclos menstruales normales y fertilidad demostrada. Este grupo de pacientes, según Botella, constituyen excepciones (mosaicos inadvertidos) y sus cariotipos que no llevan implícita la disgenesia gonadal ${ }^{3,4}$.

Nielsen plantea que la menstruación aparece de forma espontánea en aproximadamente el5 \% de las pacientes con ST, y que algunas de ellas pueden tener fertilidad normal ${ }^{16,9}$. Basándose en que estadísticamente sólo el $1 \%$ de los fetos con dotación 45X llegan a término, se ha especulado que la mayor parte de los pacientes de ST son en realidad mosaicos no detectados. Numerosos investigadores postulan que en el ST 45X la supervivencia fetal necesita de un mosaicismo, al menos, en algún órgano o tejido para poder sobrevivir ${ }^{15}$.

Otros autores plantean que en estos casos existe una mayor probabilidad de cesárea que en las embarazadas sanas, debido precisamente a que la preeclampsia, en ocasiones, obliga a interrumpir la gestación, además, de que se presentan muchos casos de desproporción cefalopélvica, explicable por la baja talla de estas embarazadas ${ }^{4}$. Estos embarazos presentan una elevada frecuencia de síndrome hipertensivo, complicaciones cardiovasculares y restricción de crecimiento intrauterino ${ }^{6}$.

Se plantea que puede existir una línea celular a nivel de los ovarios y el útero que permita el desarrollo folicular y así se logre la fecundación ${ }^{15}$

Las expectativas de fertilidad con este síndrome han cambiado en los últimos ańos gracias a los tratamientos de reproducción asistida ${ }^{8,15,13}$. Los avances en técnicas reproductivas han permitido que las mujeres portadoras de ST, puedan concebir mediante ovodonación y fertilización in vitro, realizándose el primer intento en 1984. Por lo tanto, es fundamental en todas las pacientes con ST que estén considerando un embarazo, la evaluación completa para una adecuada consejería prenatal. Durante el embarazo, se requiere monitorización estricta por equipo multidisciplinario, que incluya al menos un cardiólogo, un endocrinólogo y un especialista en medicina materno-fetal ${ }^{6}$.

En nuestro país se ha publicado un solo caso de ST y embarazo espontáneo, en el año 1998. Sugerimos diseñar estrategias para el diagnóstico precoz de esta afección con la finalidad de alcanzar una mejor calidad de vida. La incorporación al programa de reproducción asistida de estas pacientes les daría una mejor expectativa de existencia.

\section{Referencias bibliográficas}

1. Ceballos Mujica A. Función Ovárica y embarazo en pacientes con Sd Turner. SOGIA. 2013;20(1):8-13.

2. Australasian Paediatric Endocrine Group. Las hormonas y yo: Síndrome de Turner. Australia: Merck Serono Australia; 2012.

3. Botella J. Anomalías congénitas en la gónada femenina. En: Endocrinología de la mujer. 


\section{Dr. Francisco Carvajal Martínez, Dra. Danexy Borges Carcases, Dra. Yisel Ávila Baltazar, Dra. Alicia Burgos Cabrera y Dra. Yuraimi Piz Ramos}

5ed. Barcelona: Ed. Científico Médica; 1976. p. 757-62.

4. Ortiz Lee C, De Marcos N, Prieto Valdes M, Garolera Bermudez D. Mosaico Turner y embarazo. Rev cub Gynecol Obstet.1998; 24(1):24-7.

5. Fagerstrom C, Himes Pat, Olson S. Problemas en los cromosomas del sexo descubierto a través del Diagnóstico Prenatal: el Síndrome de Turner 45,X. [Internet]. 1998. Comité de Diagnóstico Prenatal de PacNoRGG [Citado 2018 Oct 10]. Diponible en tssus@turner-syndrome-us.org.2002 Folleto.

6. Burkey D, Fernandez MB, Poblete, A, Chamy F, Ogrodnik, R, Paredes AV. Embarazo por ovodonación en paciente con síndrome de Turner. Rev. Obstet. Ginecol. 2015 Nov;10(2):97-100.

7. Macedo J. El síndrome de Turner y la fertilidad (Salud y fertilidad). 2015 May 25 En Estudios de la Esterilidad Fertilidad in Vitor (GINFIV). [Internet Blog]. [Citado 2018 Sept 10]. Disponible http://www.ginefiv.com/ blog/el-sndrome-de-turner-la-fertilidad.html

8. Guerguin B, Syndrome de Turner et grossesse. Apropósito de un caso. Rev Fr Gynecol Obstet.1993;88:342-4.

9. Harold Ch. G. Atlas of genetic diagnosis and counseling. New York: Humana Press; 2006. p. 1007.

10. Velasco H, García N, Madero JI, Duque MA, Saumet J, López C. Complicaciones maternos fetales en pacientes con Síndrome Turner: Reporte de dos casos manejados con donación de óvulos. Revista Colombiana Obstetricia y Ginecología. 2006; 57(2):117-123.
11. López Siguero JP, Preguntas y respuestas sobre Síndrome de Turner. 2a ed. Madrid: Novo Nordisk Pharma, S.A; 2010.

12. Lyon Jones $\mathrm{K}$, Crandall Jones $\mathrm{M}$, Del Campo, M. Recognizable Patterns of Human Malformation. 7ed. Philadelphia: Elsevier; 2013.

13. Oktay K, Bedoschi G, Bronson R, Kashani B, McGovem P, Pal L. et al. Preservation in Womenwith Turner Syndrome: A Comprehensive Review and Practical Guidelines. J Pediatr Adolesc Gynecol. 2016 Oct;29(5):409-416. Disponible en http://doi. org/10.1016/j.jpag.2015.10.011

14. Cruz M, Bosch J. Atlas de Síndromes Pediátricos. 1ra ed. Madrid: Expax; 1998.

15. Sperling M. Pediatrics Endocrinology. 4th ed. Philadelphia: Elsevier;2014

16. Nielsen J, Sillesen I, Hansen KB. Fertility in women with Turner's Syndrome: case reportand review of literature. $\mathrm{Br} \mathrm{J}$ Obstet Gynaecol.1979;86:833-5.

17. Pasquino AM, Passeri F, PucarelliI, Segni M, Municchi G (Italian Study Group for Turner's syndrome). Spontaneous Pubertal Development in Turner's syndrome. J. Clin Endocrino/ Metab.1997;82:1810-1813.

18. Pombo M, Audí L, Bueno M, Calzada R, Carssola F, Dieguez, C. et al. Tratado de endocrinología pediátrica 4ta ed. Madrid: McGRAW-HILL Interamericana; 2009.

19. Santiago H Endocrinología en ginecología. La Habana: Editorial Ciencias Médicas; 2007. 